\title{
Performance Analysis of Fuzzy Aggregation Operations for Combining Classifiers for Natural Textures in Images
}

\author{
María Guijarro, Gonzalo Pajares, P. Javier Herrera, and J.M. de la Cruz \\ Dpt. Ingeniería del Software e Inteligencia Artificial, Facultad Informática, Universidad \\ Complutense, 28040 Madrid, Spain \\ \{mguijarro,pajares\} afdi.ucm.es, pjherrera@pdi.ucm.es, \\ jmcruz@is.ucm.es
}

\begin{abstract}
One objective for classifying pixels belonging to specific textures in natural images is to achieve the best performance in classification as possible. We propose a new unsupervised hybrid classifier. The base classifiers for hybridization are the Fuzzy Clustering and the parametric Bayesian, both supervised and selected by their well-tested performance, as reported in the literature. During the training phase we estimate the parameters of each classifier. During the decision phase we apply fuzzy aggregation operators for making the hybridization. The design of the unsupervised classifier from supervised base classifiers and the automatic computation of the final decision with fuzzy aggregation operations, make the main contributions of this paper.
\end{abstract}

Keywords: classifier combination, fuzzy aggregation, parametric estimation, fuzzy clustering, Bayes classifier.

\section{Introduction}

Nowadays the technology demands solutions for various applications. The classification of individual pixels belonging to natural textures is one of such applications due to the high spatial resolutions achieved in the images. The areas where textures are suitable include agricultural crop ordination, forest or urban identifications and damages evaluation in catastrophes or dynamic path planning during rescue missions or intervention services also in catastrophes (fires, floods).

In this work we use a pixel-based approach under the RGB color space representation because, as reported in [1], it performs favorably against other color mappings. Hence, the three RGB spectral values are the features used in our method. The same texture could be displayed with different RGB levels; this makes the problem fuzzy in nature, justifying the choice of the fuzzy aggregation operation.

Nevertheless, the main problem is: which is the best strategy for combining simple classifiers? This is an issue still open. Here we find one of the problems solved with techniques based on hybrid artificial intelligence systems [12]. Indeed in [3] it is stated that the best combination method does not exist. In [5] a revision of different approaches is reported including the way in which the classifiers are combined. Some important conclusions are: 1) if only labels are available, a majority vote should be suitable; 2) if continuous outputs like posterior probabilities are supplied, an average 
or some other linear combinations are suggested; 3) if the classifier outputs are interpreted as fuzzy membership values, fuzzy approaches could be used; 4) also it is possible to train the output classifier separately using the outputs of the input classifiers as new features. In 1) a selection criterion is applied, in 2 and 3) a fusion strategy is carried out and in 4) a hierarchical approach is used $[4,10]$.

Because we have available continuous outputs, we propose a new fusion approach which combines two base classifiers: the fuzzy clustering (FC) and the probabilistic parametric Bayesian (BP) approach [7]. The following two phases are involved: training and decision. These classifiers are selected because they provide the best performance when used in a subset of images which are to be classified. Moreover they have been broadly applied in the literature with high performances. Both FC and $\mathrm{BP}$ estimate their parameters which are stored in the Knowledge Base $(K B)$. During the classification or decision phase, each base classifier provides for each pixel a support of belonging to a cluster (FC gives membership degrees and BP probabilities). We propose the hybrid approach where the individual supports are combined through the fuzzy aggregation operations. The results are better than those obtained by the simple classifiers, as the non-parametric approach Parzen's window or the vector quantization [7]. This combined scheme, joined to the design of the unsupervised strategy, makes the main contribution to the hybrid systems.

\section{Automatic Hybrid Classifier Design}

Our system works in two stages: 1) performing a training process with a set of patterns; 2) performing a classification process, the final decision is the class that the new patterns are classified as belonging to. Figure 1 shows both processes.

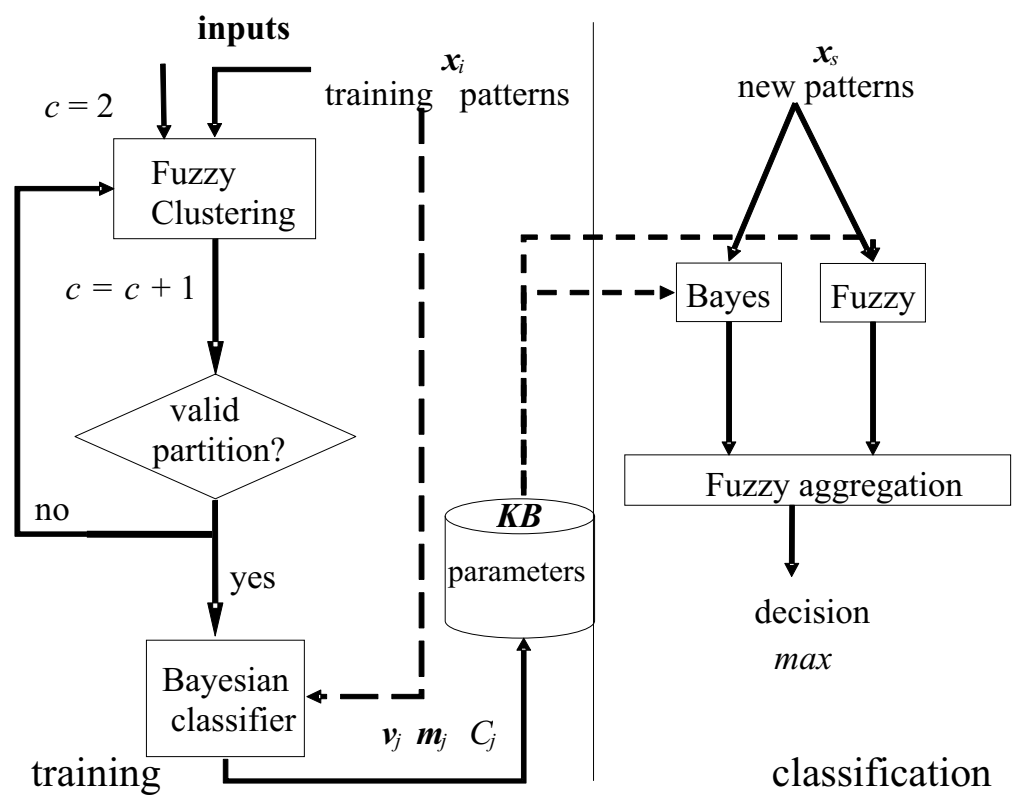

Fig. 1. Architecture of the hybrid classifier based on the fuzzy aggregation approach 
In both processes, training and classification, each pattern is characterized by a feature vector $\boldsymbol{x}$. As mentioned before, in this paper, we use a pixel-based approach and taking into account that we are classifying multiespectral textured images, we use as the attribute vector the spectral components, i.e. the red, green and blue. The RGB map performs better than other colour representations [2]. So, $\boldsymbol{x}$ is a 3-dimensional vector representing each pixel, where its components are the red, green and blue values respectively.

\section{A. The Training Process}

During the training phase, we start with the observation of a set $X$ of $n$ training patterns, i.e. $X=\left\{\boldsymbol{x}_{1}, \boldsymbol{x}_{2}, \ldots, \boldsymbol{x}_{n}\right\} \in \mathfrak{R}^{3}$. Each sample is to be assigned to a given class, where the number of possible classes is $c$. Each class is identified as $w_{j}$, where $j=1 \ldots$ $c$. Now, the problem is to assign each pattern sample to a class and compute the cluster prototypes. For such purpose we have chosen the well-tested fuzzy clustering framework which has been customized and tailored for working in an unsupervised fashion according to the criterion described in [7]. The original FC computes for each $\boldsymbol{x}_{i}$ at the iteration $k$ its membership grade and updates the cluster centers according to equation (1),

$$
\mu_{i j}(k)=\frac{1}{\sum_{r=1}^{c}\left(d_{i j}(k) / d_{i r}(k)\right)^{2 /(m-1)}} \quad \boldsymbol{v}_{j}(k+1)=\frac{\sum_{i=1}^{n} \mu_{i j}^{m}(k) \boldsymbol{x}_{i}}{\sum_{i=1}^{n} \mu_{i j}^{m}(k)}
$$

$d$ is the squared Euclidean distance, $m$ is called the exponent weight, $v$ are the cluster centers, and $\mu$ are the membership grade. The stopping criterion of the iteration process is achieved when $\left\|\mu_{i j}(k+1)-\mu_{i j}(k)\right\|<\varepsilon \forall i j$ or a number $N$ of iterations is reached. The number of classes is initially set to 2 . After the fuzzy clustering process, a partition of the input training patterns is obtained, where each cluster $j$ has associated its center $\boldsymbol{v}_{j}$. Also, for each sample $i$ its corresponding membership grades, $\mu_{i j}$, of belonging to each cluster $j$, are computed. The cluster centres are stored in the $K B$ to be recovered later.

The next step consists of the cluster validation. This is carried out by computing the partition coefficient for the number of classes specified as follows

$$
P C(U ; c)=\frac{1}{n} \sum_{i=1}^{n} \sum_{j=1}^{c}\left(\mu_{i j}\right)^{2}
$$

The maximum value of $P C$ for different values of $c$ determines the best partition, i.e. the best number of classes for the set of training samples available. Values of $P C$ near the unity indicate that the partition is acceptable. This is because the $P C$ is upper bounded by the unity.

Following the scheme in figure 1, the partition of clusters is transferred to the Bayesian classifier [7]. Under this framework the problem is reduced to compute the probability of belonging to $w_{j}$ given a sample $\boldsymbol{x}$. This parametric classifier receives the validated partition, supplied by FC and estimates the cluster centres $\boldsymbol{m}_{j}$ and the 
covariance matrices $C_{j}$ as parameters to be stored in $K B$ and recovered later. A common practice is to express the likelihood as a Gaussian function of the estimated parameters.

$$
\begin{gathered}
p\left(\boldsymbol{x} \mid w_{j}\right)=\frac{1}{(2 \pi)^{d / 2}\left|C_{j}\right|^{1 / 2}} \exp \left[-\frac{1}{2}\left(\boldsymbol{x}-\boldsymbol{m}_{j}\right)^{t} C_{j}^{-1}\left(\boldsymbol{x}-\boldsymbol{m}_{j}\right)\right] \\
\boldsymbol{m}_{j}=\frac{1}{n_{j}} \sum_{k=1}^{n_{j}} \boldsymbol{x}_{k} ; C_{j}=\frac{1}{n_{j}-1} \sum_{k=1}^{n_{j}}\left(\boldsymbol{x}_{k}-\boldsymbol{m}_{j}\right)\left(\boldsymbol{x}_{k}-\boldsymbol{m}_{j}\right)^{t}
\end{gathered}
$$

\section{B. The Classification Process: Fuzzy Aggregation Framework as a Combiner}

During the classification process new images, and consequently new texture patterns, are to be processed by the system. With such purpose, we recover the $\boldsymbol{v}_{j}$ cluster centers, the covariance matrices $C_{j}$ and the $\boldsymbol{m}_{j}$ mean clusters, which were stored in $K B$ during the training process. The original Bayes classifier includes the a priori probability to be combined with the likelihood in the computation of the a posterior probability, but given a pixel to be classified we do not know nothing about its assignment to the clusters, therefore we must assume that a priori, before the observation, all pixels have identical a priori probabilities. Therefore the a priori probability is not discriminant, it can be avoided and the decision can be made only based on the likelihoods estimated according to equation (3).

The fuzzy logic framework provides a number of functions for aggregating two or more fuzzy sets. In this paper, we will introduce these functions and give details about how to use them during classification phase.

The combination is carried out taking into account the supports provided by the selected classifiers, $F C$ and $B P . F C$ and $B P$ provide respectively as supports the membership degree $\mu_{j}(\boldsymbol{x})$ and probability $p_{j}(\boldsymbol{x})$ that a pattern $\boldsymbol{x}$ belongs to a cluster $c_{j}$. Assuming that $p_{j}(\boldsymbol{x})$ is a fuzzy magnitude ranging in $[0,1]$ we combine both supports through the aggregation operations defined below. Given the pattern sample $\boldsymbol{x}$ with the supports provided by the simple classifiers $\mu_{j}(x)$ and $p_{j}(x)$ the combination is carried out based on the fuzzy aggregation operations from (4) to (15) [8,9]. After experimentation, in this paper the $\gamma$ parameters (equations $\mathrm{HI}$ and $\mathrm{HU}$ ) and $r$ (equations YI and YU) are set to 2, and $\alpha$ (equations DPI and DPU) and $\gamma$ (equations $\mathrm{WI}$ and WU) are set to 0.5 .

Hamacher Intersection (HI) and Hamacher Union (HU):

$$
\begin{gathered}
d_{j}(\boldsymbol{x})=\frac{\mu_{j}(\boldsymbol{x}) p_{j}(\boldsymbol{x})}{\gamma+(1-\gamma)\left(\mu_{j}(\boldsymbol{x})+p_{j}(\boldsymbol{x})-\mu_{j}(\boldsymbol{x}) p_{j}(\boldsymbol{x})\right)} \\
d_{j}(\boldsymbol{x})=\frac{(\gamma-1) \mu_{j}(\boldsymbol{x}) p_{j}(\boldsymbol{x})+\mu_{j}(\boldsymbol{x})+p_{j}(\boldsymbol{x})}{1+\gamma \mu_{j}(\boldsymbol{x}) p_{j}(\boldsymbol{x})}
\end{gathered}
$$

Yager Intersection (YI) and Yager Union (YU): 


$$
\begin{gathered}
d_{j}(\boldsymbol{x})=1-\min \left\{1,\left(\left(1-\mu_{j}(\boldsymbol{x})\right)^{r}+\left(1-p_{j}(\boldsymbol{x})\right)^{r}\right) \frac{1}{r}\right\} \\
d_{j}(\boldsymbol{x})=\min \left\{1,\left(\left(\mu_{j}(\boldsymbol{x})\right)^{r}+\left(p_{j}(\boldsymbol{x})\right)^{r}\right)^{\frac{1}{r}}\right\}
\end{gathered}
$$

Dubois and Prade Intersection (DPI) and Dubois and Prade Union (DPU):

$$
\begin{gathered}
d_{j}(\boldsymbol{x})=\frac{\mu_{j}(\boldsymbol{x}) p_{j}(\boldsymbol{x})}{\max \left\{\mu_{j}(\boldsymbol{x}), p_{j}(\boldsymbol{x}), \alpha\right\}} \\
d_{j}(\boldsymbol{x})=\frac{\mu_{j}(\boldsymbol{x})+p_{j}(\boldsymbol{x})-\mu_{j}(\boldsymbol{x}) p_{j}(\boldsymbol{x})-\min \left\{\mu_{j}(\boldsymbol{x}), p_{j}(\boldsymbol{x}),(1-\alpha)\right\}}{\max \left\{\left(1-\mu_{j}(\boldsymbol{x})\right),\left(1-p_{j}(\boldsymbol{x})\right), \alpha\right\}}
\end{gathered}
$$

Werners Intersection (WI) and Werners Union (WU):

$$
\begin{gathered}
d_{j}(\boldsymbol{x})=\gamma \min \left\{\mu_{j}(\boldsymbol{x}), p_{j}(\boldsymbol{x})\right\}+\frac{(1-\gamma)\left(\mu_{j}(\boldsymbol{x})+p_{j}(\boldsymbol{x})\right)}{2} . \\
d_{j}(\boldsymbol{x})=\gamma \max \left\{\mu_{j}(\boldsymbol{x}), p_{j}(\boldsymbol{x})\right\}+\frac{(1-\gamma)\left(\mu_{j}(\boldsymbol{x})+p_{j}(\boldsymbol{x})\right)}{2}
\end{gathered}
$$

Symmetric Summations M1 (M1) and Symmetric Summations M2 (M2):

$$
\begin{aligned}
& d_{j}(\boldsymbol{x})=\frac{\mu_{j}(\boldsymbol{x})+p_{j}(\boldsymbol{x})-\mu_{j}(\boldsymbol{x}) p_{j}(\boldsymbol{x})}{1+\mu_{j}(\boldsymbol{x})+p_{j}(\boldsymbol{x})-2 \mu_{j}(\boldsymbol{x}) p_{j}(\boldsymbol{x})} \\
& d_{j}(\boldsymbol{x})=\frac{\mu_{j}(\boldsymbol{x}) p_{j}(\boldsymbol{x})}{1-\mu_{j}(\boldsymbol{x})-p_{j}(\boldsymbol{x})+2 \mu_{j}(\boldsymbol{x}) p_{j}(\boldsymbol{x})}
\end{aligned}
$$

Symmetric Differences N1 (N1) and Symmetric Differences N2 (N2):

$$
\begin{aligned}
& d_{j}(\boldsymbol{x})=\frac{\max \left\{\mu_{j}(\boldsymbol{x}), p_{j}(\boldsymbol{x})\right\}}{1+\left|\mu_{j}(\boldsymbol{x})-p_{j}(\boldsymbol{x})\right|} \\
& d_{j}(\boldsymbol{x})=\frac{\min \left\{\mu_{j}(\boldsymbol{x}), p_{j}(\boldsymbol{x})\right\}}{1-\left|\mu_{j}(\boldsymbol{x})-p_{j}(\boldsymbol{x})\right|}
\end{aligned}
$$

The final decision for the sample $\boldsymbol{x}$ in each of these fuzzy aggregation operations is made by choosing the maximum of $w_{j}$ obtained, through the following expression,

$$
\boldsymbol{x} \in w_{j} \quad \text { if } \quad\left(d_{j}\right)>\left(d_{k}\right) \forall d_{k} \mid d_{k} \neq d_{j}
$$




\section{Comparative Analysis and Performance Evaluation}

To assess the validity and performance of the proposed approach we describe the tests carried out according to both processes: training and classification.

We have used a set of 26 digital aerial images acquired during May in 2006 from the Abadin region located at Lugo (Spain). They are multispectral images with $512 \times 512$ pixels in size. The images are taken at different days from an area with several natural textures. The initial training patterns are extracted from 10 images of the full set. The remainder 16 images are used for testing. The images assigned to each set are randomly selected from the 26 images available.

\subsection{Unsupervised Training: Estimating the Best Partition}

The first goal is to determine the number of classes that will validate the initial partition [12]. Figure 2 shows the behavior of the partition coefficient, $P C$ equation (2), versus the number of clusters. It should be noted that there are two partition coefficient values exceeding the threshold value of 0.8 in our experiments, considered as appropriate. These correspond to the values of $c$ for 4 and 5, with respective values of 0.86 and 0.82 . As the maximum value is obtained for $c=4$, this value is eventually chosen as the number of clusters for classifying our images. The reminder clusters do not achieve acceptable values according to criteria set threshold 0.8 .

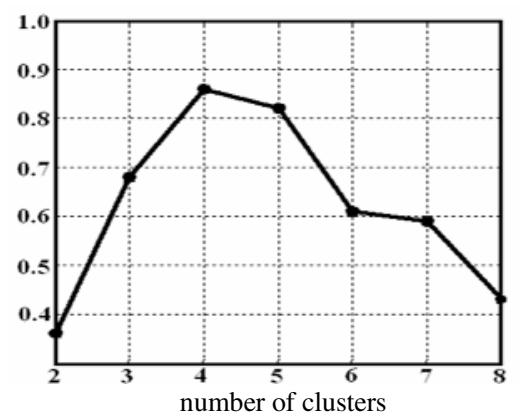

Fig. 2. Values for PC against the number of clusters

\subsection{Design of a Test Strategy}

The set of 26 images is split into two subsets $A$ and $B$ with ten and sixteen images respectively; an initial training process is carried out with $A$ using FC and BP. The set $B$ is classified after this initial training phase. The above mentioned parameters estimated by FC and BP are stored in $K B$. During the classification phase the new patterns are classified by $\mathrm{FC}$ and $\mathrm{BP}$ and also through the aggregations defined in equations (4) to (15) recovering the parameters stored in $K B$. To verify the performance of the base, FC, BP, and combined, equations (4) to (15), classifiers we build a ground truth for each image under the supervision of the expert human criterion considering that the number of clusters is four as obtained based on the $P C$ coefficient, which agree with the expert criterion. For each class we build a binary 
image, which is manually touched up until a satisfactory classification is obtained under the human supervision. Using the ground truth for each simple classifier we choose two of the best results, which are the fuzzy clustering classifier and the Bayesian classifier.

In summary, the full test process is carried out according to the following steps:

STEP 0 (initial training): for each image (from the 26 available) we perform a downsampling by 4 , i.e. we obtain $26 \times 128 \times 128$ training samples.

For $c=2$ to $c=8$ (maximum number of classes allowed) validate the partition by computing the partition coefficient $P C$ through the equation (2) and determine the best partition (number of classes and centers). These classes are used for classifying the pattern samples during the next steps.

STEP 1: given the images in A, classify each pixel as belonging to a textured class, which has been identified previously, according to the FC and BC. Store the parameters in $\mathrm{KB}$ to be used in the next step.

STEP 2: using the set $\mathrm{B}$, classify it according to $\mathrm{FC}$ and $\mathrm{BC}$ and also make a decision with the different fuzzy aggregation operations. Compute the percentage of successes according to the ground truth defined for each class at each image.

\subsection{Analysis of Results}

In order to verify the performance of the proposed hybrid strategy, we compare the percentage of error obtained during the classification phase for the base classifiers and the hybrid ones according to the ground truth and the four classes estimated as valid. Table 1 shows these percentages.

Table 1. Percentage of error obtained for the methods analysed

\begin{tabular}{|c|c|c|c|c|c|c|c|c|c|c|c|c|}
\hline \multirow[t]{2}{*}{ Base } & FC & $\mathbf{B P}$ & & & & & & & & & & \\
\hline & 17.04 & 17.83 & & & & & & & & & & \\
\hline \multirow[b]{2}{*}{ Hybrid } & HU & HI & YU & YI & DPU & DPI & WU & \begin{tabular}{|l|l} 
WI \\
\end{tabular} & \begin{tabular}{|l|} 
M1 \\
\end{tabular} & \begin{tabular}{|l|} 
M2 \\
\end{tabular} & N1 & N2 \\
\hline & $\overline{15.42}$ & 16.68 & 17.02 & 17.10 & 16.13 & 16.52 & 16.63 & 16.79 & 16.94 & 16.94 & 31.00 & 19.39 \\
\hline
\end{tabular}

From results in table 1, one can see that the error obtained with FC is comparable to that obtained with BP. Also that the best performances are obtained with the fuzzy aggregation operations, like HU, HI, DPU, DPI, WU, WI, M1 and M2. The best and worse performances are obtained by $\mathrm{HU}$ and N1 respectively. We can infer as a general conclusion that the hybridization improves the classification results. Exception made with N1 and N2. Figure $3(a)$ displays an original image which is to be classified; $(b)$ displays the correspondence between the classes and the color assigned to the corresponding cluster center according to a color map previously defined; $(c)$ labeled image for the four clusters obtained by the hybrid HU approach. The correspondence between labels and the different textures is as follows, 1: yellow with forest vegetation, 2: blue with bare soil, 3: green with agricultural crop vegetation, 4: red with buildings and man made structures. 


\section{Conclusions}

We propose a new hybrid classifier for natural textures. The proposed method combines two classifiers, FC and BP through fuzzy aggregation operations. The performance of the hybrid approach is compared against the base classifiers, verifying that it performs favourably in the set of aerial images tested. This approach could be applicable to other textured images even with different attributes. Also, as a future work different number of classifiers could be used by applying the associative property of fuzzy aggregations. Moreover, thanks to the proposed design, the method becomes unsupervised even though the base classifiers are supervised.
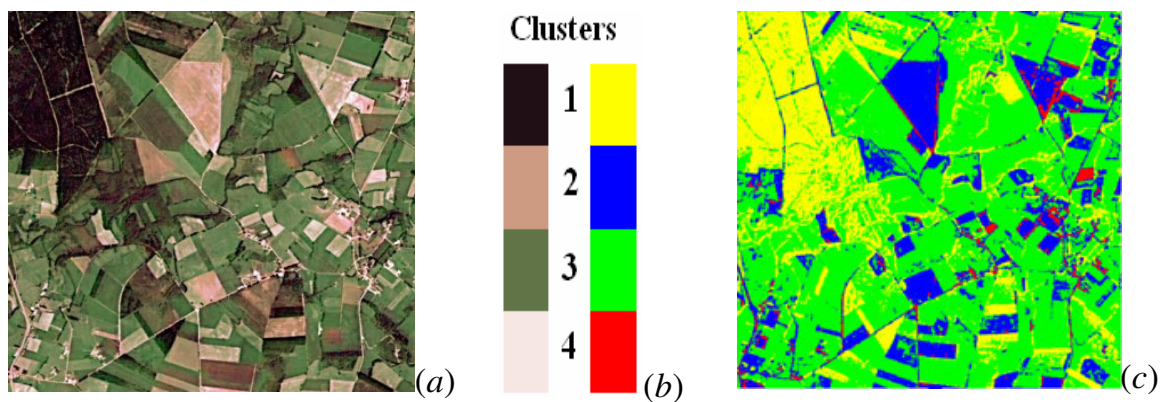

Fig. 3. (a) Original image; (b) colors and labels; (c) labeled image result of the HU

Acknowledgments. Partial funding has also been received from DPI2009-14552C02-01 project, supported by the Ministerio de Educación y Ciencia of Spain within the Plan Nacional de I+D+i.

\section{References}

1. Drimbarean, P.F., Whelan, P.F.: Experiments in Colour Texture Analysis. Pattern Recognition Letters 22, 1161-1167 (2003)

2. Kittler, J., Hatef, M., Duin, R.P.W., Matas, J.: On Combining Classifiers. IEEE Trans. on Pattern Analysis and Machine Intelligence 20(3), 226-239 (1998)

3. Kuncheva, L.I.: Combining Pattern Classifiers: Methods and Algorithms. Wiley, Chichester (2004)

4. Valdovinos, R.M., Sánchez, J.S., Barandela, R.: Dynamic and Static Weighting in Classifier Fusion. In: Marques, J.S., Pérez de la Blanca, N., Pina, P. (eds.) IbPRIA 2005. LNCS, vol. 3523, pp. 59-66. Springer, Heidelberg (2005)

5. Duda, R.O., Hart, P.E., Stork, D.S.: Pattern Classification. Wiley, Chichester (2000)

6. Zimmermann, H.J.: Fuzzy Set Theory and its Applications. Kluwer Academic Publishers, Norwell (1991)

7. Guijarro, M., Pajares, G., Abreu, R.: A New Unsupervised Hybrid Classifier for Natural Textures in Images. In: HAIS, vol. 44, pp. 280-287. Springer, Heidelberg (2007)

8. Derrac, J., García, S., Herrera, F.: A First Study on the Use of Coevolutionary Algorithms for Instance and Feature Selection. In: Corchado, E., Wu, X., Oja, E., Herrero, Á., Baruque, B. (eds.) HAIS 2009. LNCS, vol. 5572, pp. 557-564. Springer, Heidelberg (2009) 
9. Corchado, E., Abraham, A., Carvalho, A.C.P.L.F.D.: Hybrid intelligent algorithms and applications. Information Science 180(14), 2633-2634 (2010)

10. Wozniak, M., Zmyslony, M.: Designing Fusers on the Basis of Discriminants Evolutionary and Neural Methods of Training. HAIS 1, 590-597 (2010)

11. Abraham, A., Corchado, E., Corchado, J.M.: Hybrid learning machines. Neurocomputing 72(13-15), 2729-2730 (2009) 